\title{
Apuesto a que toma tamsulosina
}

\section{I guess you take tamsulosin}

Presentamos el caso de un paciente varón de 71 años de edad que acude para operarse de catarata de su ojo derecho. Como antecedentes personales, destacar que es diabético tipo II desde hace 4 años. Sigue tratamiento con diamicron y adiro, y una pastilla diaria para la próstata cuyo nombre no recuerda.

La agudeza visual es 0,3 en su ojo derecho y 0,6 en su ojo izquierdo. El examen en la lámpara de hendidura, demuestra la presencia de una catarata nuclear en ambos ojos, algo más avanzada en su ojo derecho que justifican su mala agudeza visual. El fondo de ojo es normal, no presentando retinopatía diabética en este momento. Por todo ello el paciente es incluido en lista de espera para llevar a cabo una facoemulsificación de la catarata de su ojo derecho con implante de lente intraocular.

En la unidad de cirugía mayor ambulatoria es sometido al protocolo habitual de dilatación pupilar, que incluye la aplicación de 3 gotas de tropicamida y 3 gotas de fenilefrina, haciéndose evidente una muy mala midriasis, que obliga a la utilización de ganchos de iris durante la cirugía (fig. 1). La cirugía se lleva a cabo utilizando anestesia subtenoniana, pues la manipulación del iris puede ser molesta bajo anestesia tópica, dura unos $45 \mathrm{~min}$, tiempo superior al habitual y se finaliza sin complicaciones, aunque durante el procedimiento se hace evidente la gran flacidez del iris y su tendencia constante a herniarse a través de las incisiones corneales.

En el año 2005 Chang describió por primera vez un síndrome, que denominó síndrome del iris flácido intraoperatorio ${ }^{1}$. Este síndrome se caracteriza por la asociación de flacidez iridiana, tendencia al prolapso del iris a través de las incisiones corneales y pérdida progresiva de la midriasis farmacológica a lo largo de la cirugía de catarata, y se ha relacionado con el consumo crónico de antagonistas adrenérgicos, y de forma muy especial con el consumo de tamsulosina.

Este síndrome se ha descrito también en relación con otros antagonistas alfa como terazosina, doxazosina, alfuzosina, prazosín o indoramina ${ }^{2}$, antagonistas de los receptores beta como el labetalol o incluso con fármacos de otros grupos (antipsicóticos como el zuclopenthixol, antidepresivos como la mianserina, vasopresores, inhibidores de la 5 alfa reductasa como el finasteride o incluso con remedios de herbolario como el extracto de saw palmetto) ${ }^{3}$. Sin embargo, lo cierto es que la mayor parte de la literatura pone de manifiesto que la relación es especialmente estrecha con la tamsulosina. Uno de los trabajos mencionados, estudiaba 92 ojos y encontraba un OR de 32,15 entre tamsulosina y alfuzosina a favor de la primera ${ }^{2}$.

La tamsulosina es un fármaco antagonista de los receptores alfa adrenérgicos, aprobado por la FDA para tratar los síntomas producidos por la hipertrofia benigna de próstata en varones, aunque también se usa de forma compasiva en el tratamiento de la retención urinaria en mujeres. En España existen al menos de 10 presentaciones comerciales. El fármaco se comercializa en 2 formulaciones, una de ellas tiene una vida media de aproximadamente $12 \mathrm{~h}$, en tanto que existe otra formulación de liberación retardada que tiene una vida media de aproximadamente $24 \mathrm{~h}$.

La razón fisiopatológica por la que este efecto secundario es tan específico de esta droga, es que la tamsulosina inhibe de forma selectiva los receptores alfa 1A de la próstata, y gracias a esta especificidad produce muy poca hipotensión ortostática. Sin embargo estos receptores alfa 1A son los receptores adrenérgicos mayoritarios en el músculo dilatador de la pupila. El bloqueo continuo de estos receptores conduce a la atrofia del citado músculo. Ello condiciona la mala midriasis inicial y la tendencia a la salida del iris a través de las incisiones. Por todo ello el tiempo quirúrgico se prolonga de forma significativa y aumenta la probabilidad de que se produzcan complicaciones (fundamentalmente rotura de cápsula posterior, desinserción zonular, atrofia traumática del iris, picos hipertensivos postquirúrgicos y descompensación corneal por pérdida endotelial) ${ }^{2}$.

Toda la literatura publicada al respecto coincide en que el consumo de tamsulosina aumenta de forma muy significativa la probabilidad de que se produzcan complicaciones durante la cirugía de catarata (y en menor medida durante otras cirugías oftalmológicas como la cirugía de glaucoma). Es difícil dimensionar exactamente el problema, pues no todos los pacientes que toman tamsulosina desarrollan un síndrome de iris flácido durante la cirugía de catarata, ni todos lo desarrollan con la misma severidad. Aún así de lo que no hay duda es de que se trata de un problema epidemiológico muy relevante, pues las 2 patologías que condicionan su aparición son muy prevalentes. Por ello recientemente este síndrome ha salido de la literatura oftalmológica y ha llegado a ocupar un editorial en la revista $\mathrm{JAMA}^{4}$. Esta misma revista publicaba en ese mismo número un riguroso trabajo, en el que los autores tras analizar 96.128 procedimientos quirúrgicos en el área sanitaria de Ontario, llegaban a la conclusión de que el hecho de que consumir tamsulosina multiplicaba por 2,33 el riesgo de que el paciente tuviera que ser sometido en el postoperatorio inmediato a una segunda cirugía ${ }^{4}$ Teniendo en cuenta que la mayor parte de los pacientes que sufren una complicación no precisan una segunda cirugía, podemos hacernos una idea de las dimensiones que alcanza este problema. Este $2,33 \%$ no es sino la punta de un gran iceberg que permanece en gran medida oculto.

Pronto se vio que no se trataba de un efecto secundario farmacológico en el sentido clásico del término, pues el síndrome puede producirse varios años después de haber cesado el tratamiento. Este carácter al menos parcialmente irreversible se cree debido a que la inhibición continuada por parte de la droga del músculo dilatador de la pupila conduce a la atrofia del mismo. De hecho, recientemente se ha demostrado utilizando tomografía óptica de coherencia 

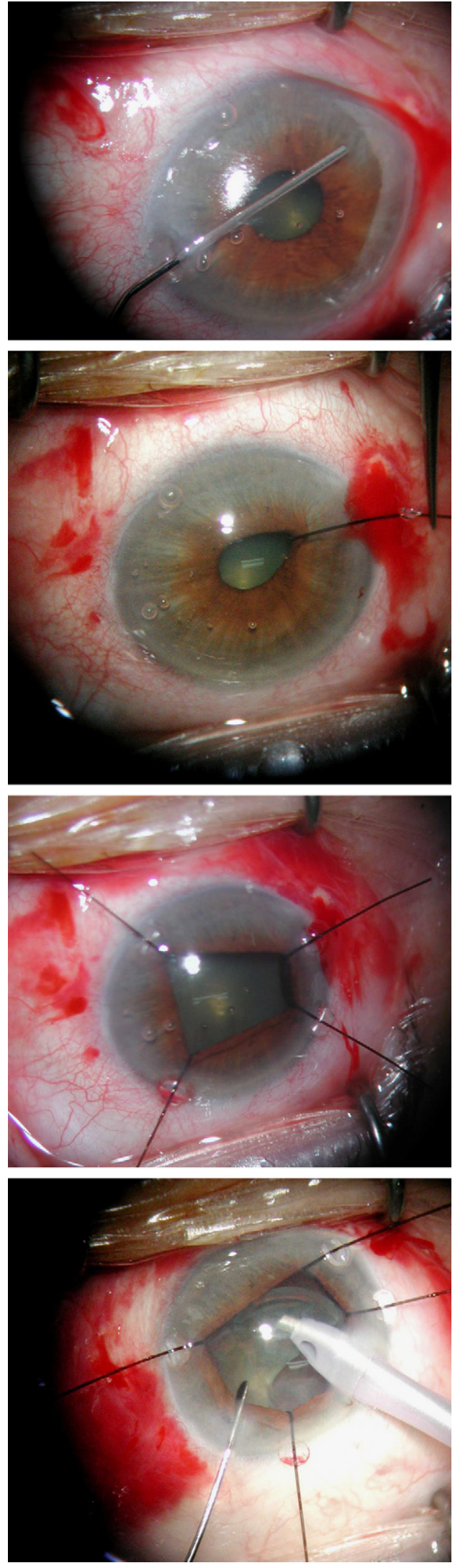

Figura 1 - Secuencia que resume la cirugía de catarata en un paciente que consumía tamsulosina. Como consecuencia de la mala midriasis fue necesaria la utilización de retractores de iris.
(OCT) del segmento anterior que los pacientes que consumen o han consumido fármacos antagonistas de los receptores alfa presentan una reducción muy significativa del espesor iridiano (355 micras en el grupo tratado, 447 micras en el grupo control) $)^{5}$. Por ello retirar el fármaco unos días antes de la cirugía no esta claro que resulte totalmente eficaz.

Aunque algunos autores abogan por premedicar al paciente con atropina o inyectar epinefrina en la cámara anterior durante la cirugía, lo cierto es que no existe consenso acerca de si estas medidas resultan eficaces ${ }^{6-8}$, y en la mayor parte de las ocasiones el cirujano se ve obligado a emplear dispositivos mecánicos, para mantener una midriasis adecuada a lo largo de la cirugía. Los más populares son los ganchos de iris (fig. 1), pero hay también otros comercializados como el anillo de Malyugin ${ }^{8,9}$.

Creemos que el problema comienza no el día que el paciente se opera de catarata, sino varios años antes cuando el urólogo prescribe tamsulosina a un paciente fáquico (no operado de catarata). Por ello resulta vital que los especialistas en urología conozcan este síndrome. Creemos que el hecho de que el paciente sea fáquico, debe ser considerado una contraindicación relativa para iniciar el tratamiento con tamsulosina, y que en caso de ser necesario, el paciente debería ser sometido a una valoración oftalmológica previa ${ }^{10}$.

B I B L I O G R A F Í A

1. Chang DF, Campbell JR. Intraoperative floppy iris syndrome associated with tamsulosin. J Cataract Refract Surg. 2005;31: 664-73.

2. Blouin MC, Blouin J, Perreault S, Lapointe A, Dragomir A. Intraoperative floppy-iris syndrome associated with alpha1adrenoreceptors: Comparison of tamsulosin and alfuzosin. J Cataract Refract Surg. 2007;33:1227-34.

3. Pringle E, Packard R. Antipsychotic agent as an etiologic agent of IFIS. J Cataract Refract Surg. 2005;31:2240 2241, author reply 2241.

4. Bell CM, Hatch WV, Fischer HD, Cernat G, Paterson JM, Gruneir A, et al. Association between tamsulosin and serious ophthalmic adverse events in older men following cataract surgery. JAMA. 2009;301:1991-6.

5. Prata TS, Palmiero PM, Angelilli A, Sbeity Z, De Moraes CG, Liebmann JM, et al. Iris morphologic changes related to alpha(1)-adrenergic receptor antagonists implications for intraoperative floppy iris syndrome. Ophthalmology. 2009;116: 877-881.

6. Shugar JK. Prophylaxis for IFIS. J Cataract Refract Surg. 2007; 33:942-3.

7. Bendel RE, Phillips MB. Preoperative use of atropine to prevent intraoperative floppy-iris syndrome in patients taking tamsulosin. J Cataract Refract Surg. 2006;32:1603-5.

8. Chang DF, Braga-Mele R, Mamalis N, Masket S, Miller KM, Nichamin LD, et al. ASCRS white paper: Clinical review of intraoperative floppy-iris syndrome. J Cataract Refract Surg. 2008;34:2153-62.

9. Chang DF, Braga-Mele R, Mamalis N, Masket S, Miller KM, Nichamin LD, et al. Clinical experience with intraoperative floppy-iris syndrome results of the 2008 ASCRS member survey. J Cataract Refract Surg. 2008;34:1201-9.

10. Gonzalez Martin-Moro J, Martinez Silva V. Treatment with tamsulosin. Should the cataract be operated before? Arch Esp Urol. 2008;61:921-2. 
J. González Martín-Moro ${ }^{a, *}$, D. Santos Arrontes ${ }^{b}$,

C. Izquierdo Rodríguez ${ }^{a}$ y E. Dorronzoro Ramírez ${ }^{a}$

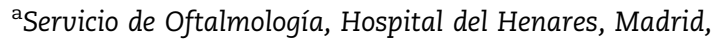
España bervicio de Urología, Hospital del Henares, Madrid, España

*Autor para correspondencia.

Correo electrónico: juliogmm@yahoo.es

(J. González Martín-Moro).On-line el 24 de abril de 2010

\section{Enfermedad de Peyronie. Escisión completa de la placa e injerto autólogo con fascia anterior de los rectos}

\section{Peyronie's disease. Complete plaque excision and autologous graft with anterior rectus fascia}

\section{Sr. Director:}

Presentamos el caso de un paciente varón de 52 años, con antecedentes personales de diabetes tipo 2, HTA, accidente isquémico transitorio cerebral, disfunción eréctil en tratamiento con inhibidores de la fosfodiestearasa, que consultó por presentar desde hacía 2 años una incurvación dorsal dolorosa del pene que había aumentado a pesar del tratamiento médico con vitamina E+colchicina y que le dificultaba prácticamente la penetración. En la exploración se palpó una placa densa, fibrosa, localizada en la línea media dorsal a nivel de la base del pene, de aproximadamente $3 \times 1,5 \mathrm{~cm}$. Realizamos el test de Kelami con el que se calcularon los grados de incurvación $\left(45^{\circ}\right)$ y una ecografía peneana, que confirmó el diagnóstico y el tamaño de la placa. Durante el procedimiento quirúrgico, y tras realizar la erección artificial, observamos que el grado de incurvación era algo mayor $\left(55^{\circ}\right)$ y que el tamaño del pene era limitado ( $12 \mathrm{~cm}$ en erección), por lo que realizamos escisión completa de la placa con bisturí frío+injerto autólogo de fascia anterior de los rectos+plicatura ventrolateral de la albugínea (figs. 1 y 2). Posteriormente se procedió a la colocación de una malla de polipropileno para

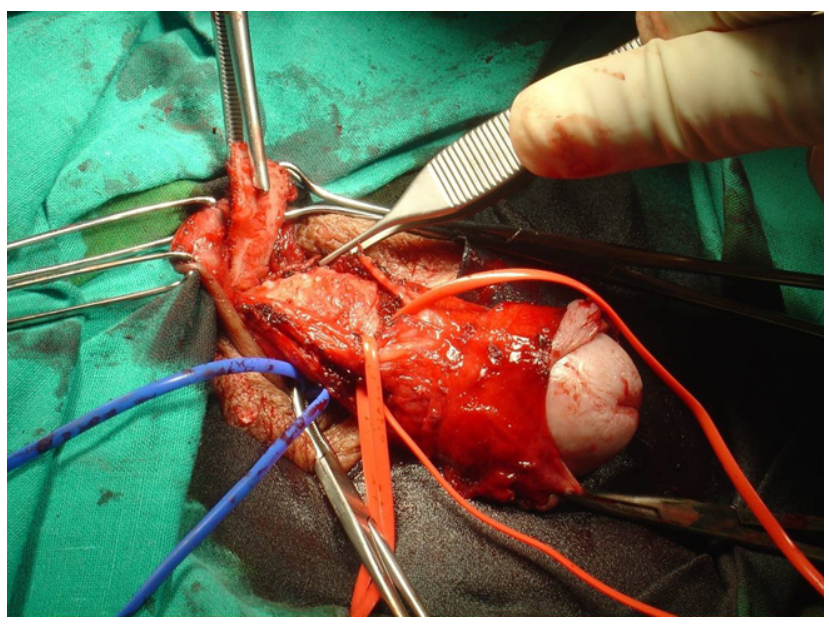

Figura 1 - Liberación de placa de Peyronie con bisturí frío. cerrar el defecto de la fascia anterior. A los 12 meses postcirugía, el paciente presenta erecciones con inhibidores de la fosfodiestearasa (de los cuales precisaba previamente), sensibilidad conservada e incurvación mínima que no le dificulta el coito.

La enfermedad de Peyronie es una enfermedad poco común, probablemente consecuencia de traumatismos a nivel del pene (aunque su etiología es aún incierta) que originan una inflamación de la túnica albugínea y, en última instancia, se produce la cicatrización y la incurvación de este $^{1-3}$. El tratamiento inicial de la presentación aguda de la enfermedad es conservador, siendo la vitamina $E$, la colchicina, los antiinflamatorios no esteroideos, etc., los fármacos comúnmente utilizados ${ }^{2}$. El tratamiento quirúrgico queda generalmente reservado para los casos que no responden al tratamiento conservador, una vez estabilizado el proceso de fibrosis, y para los pacientes con graves deformidades en el pene que le impidan una relación sexual satisfactoria ${ }^{4}$. Las opciones quirúrgicas varían en función del tamaño de la placa, la longitud del pene, el grado de

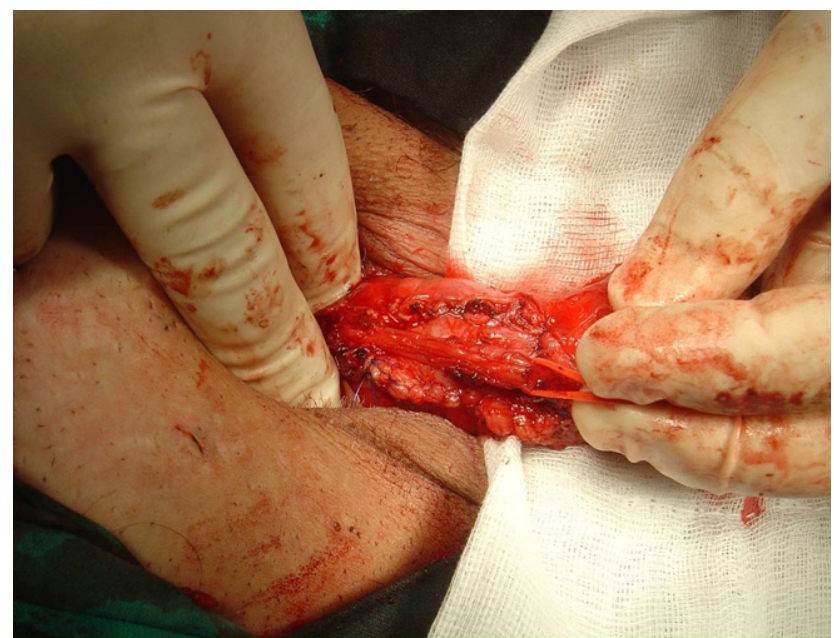

Figura 2 - Colocación de injerto sobre los cuerpos cavernosos y fijación con dos suturas continuas de vicryl 4/0. 